\title{
Computing All Power Integral Bases of Cubic Fields
}

\author{
By I. Gaál* and N. Schulte
}

\begin{abstract}
Applying Baker's effective method and the reduction procedure of Baker and Davenport, we present several lists of solutions of index form equations in (totally real and complex) cubic algebraic number fields. These solutions yield all power integral bases of these fields.
\end{abstract}

1. Introduction. Let $K$ be a cubic algebraic number field and denote by $Z_{K}$ the ring of integers of $K$. A power integral basis of $K$ is an integral basis of the form $\left\{1, \alpha, \alpha^{2}\right\}$ with some $\alpha \in Z_{K}$. If there exists such an $\alpha$, then we say that $K$ is monogenic, since $Z_{K}=Z[\alpha]$. Obviously, if $\alpha$ has this property, then it holds also for $\alpha+k$ with any $k \in Z$.

From a practical point of view, it is important to know whether there exists a power integral basis of $K$, and if so, what are the numerical values of $\alpha$.

If the Galois group of $K$ is cyclic, then the discriminant of $K$ is a full square. The problem of monogeneity in cyclic cubic fields was considered by M. N. Gras [12], [13], Archinard [1] and Dummit and Kisilevsky [4]. M. N. Gras and Archinard gave necessary and sufficient conditions for monogeneity and tested for several numerical examples whether or not the field is monogenic. Moreover, Dummit and Kisilevsky proved that there exist infinitely many cyclic cubic fields with power integral bases.

For arbitrary algebraic number fields $L$, it was proved by Györy [14] that up to obvious translations by elements of $Z$, there are only finitely many $\alpha \in Z_{L}$ with $Z_{L}=Z[\alpha]$, and he gave effective (but rather large) bounds for the sizes of these $\alpha$.

In this paper we present a method which allows us to determine all possible values of $\alpha$ (up to translation with rational integers) such that $\left\{1, \alpha, \alpha^{2}\right\}$ is a power integral basis of a given cubic number field $K$. Let $\left\{w_{1}=1, w_{2}, w_{3}\right\}$ be an integral basis of $K$. Denote by $\beta^{(i)}(i=1,2,3)$ the conjugates of any $\beta \in K$. The discriminant of the linear form $w_{2} X+w_{3} Y$ can be written as

$$
\begin{aligned}
D_{K / Q}\left(w_{2} X+w_{3} Y\right) & =\prod_{1 \leq i<j \leq 3}\left(\left(w_{2}^{(i)}-w_{2}^{(j)}\right) X+\left(w_{3}^{(i)}-w_{3}^{(j)}\right) Y\right)^{2} \\
& =(I(X, Y))^{2} D,
\end{aligned}
$$

Received August 13, 1988.

1980 Mathematics Subject Classification (1985 Revision). Primary 11Y50; Secondary $11 D 57$.

Key words and phrases. Computer solution of Diophantine equations, index form equation, Thue equation, Davenport's lemma, power integral bases.

${ }^{*}$ Research supported in part by the Hungarian National Foundation for Scientific Research, Grant No. 273/86. 
where $D$ is the discriminant of the field $K$ and $I(X, Y)$ is a homogeneous cubic polynomial with rational integral coefficients, which is called the index form with respect to the basis $\left\{w_{1}=1, w_{2}, w_{3}\right\}$ of $K$. The equation above yields that $\left\{1, \alpha, \alpha^{2}\right\}$ $\left(\alpha=x w_{2}+y w_{3}\right)$ is an integral basis of the field $K$ if and only if $D_{K / Q}(\alpha)=D$, that is, if $(x, y)$ is a solution of the index form equation

$$
I(x, y)= \pm 1 \quad(x, y \in Z) .
$$

In the special case of cubic number fields, the index form equation (1) is just a cubic Thue equation. Using Baker's method, effective bounds for the solutions of index form equations (corresponding to arbitrary algebraic number fields) were obtained by Györy [14]. His result was later improved and generalized by Györy and Papp [17], Trelina [25] and Györy [15], [16]. These bounds imply, in principle, that the solutions can be determined, but the bounds are too high for practical applications. On the other hand, an idea of Baker and Davenport [2] makes it possible to reduce the large upper bounds. Applying this method, Ellison [7], Ellison et al. [8], Steiner [24] and Pethö and Schulenberg [23] solved completely certain Thue equations of degree three and four. Moreover, Pethö [21] worked out a fast method to find "small" solutions of Thue equations.

2. Brief Sketch of the Algorithm. Besides new ideas, our method also involves some standard arguments (used by the authors quoted above). We therefore do not go into details describing those algorithms; we shall only recall the main steps of our algorithm, point out the differences between the real and complex cases and stress the new features in comparison with equations solved by other authors.

Let the index form equation (1) (corresponding to the integral basis $\left\{1, w_{2}, w_{3}\right\}$ ) of the cubic field $K$ be

$$
I(x, y)=I_{3} x^{3}+I_{2} x^{2} y+I_{1} x y^{2}+I_{0} y^{3}= \pm 1 \quad(x, y \in Z) .
$$

Denote by $\tau$ a root of $I(x, 1)=0$. We remark that $\tau$ can be chosen to generate (the same field) $K$ over $Q$. Let $x, y \in Z$ be an arbitrary but fixed solution of (2) and put $\beta=I_{3}(x-\tau y)$. $\beta$ is an integer of $K$, and (2) can be written as

$$
\beta^{(1)} \beta^{(2)} \beta^{(3)}= \pm I_{3}^{2} \text {. }
$$

In the real case, denote by $\eta_{1}, \eta_{2}$ (resp. by $\eta_{1}$ in the complex case) the fundamental units of $K$ with norm +1 . Then we set

$$
\beta=\gamma \eta_{1}^{b_{1}} \eta_{2}^{b_{2}} \quad\left(\operatorname{resp} . \beta=\gamma \eta_{1}^{b_{1}}\right)
$$

where $b_{1}, b_{2} \in Z$ (resp. $b_{1} \in Z$ ) and $\gamma$ is an integral element in $K$ with norm $\pm I_{3}^{2}$. If $\left|I_{3}\right| \neq 1$, then in the sequel we consider separately each element of a full set of nonassociate elements $\gamma$ of norm $I_{3}^{2}$. Such a set can be determined, e.g., by the method of Fincke and Pohst [10]. We remark that this is the first time that Thue equations with $\left|I_{3}\right| \neq 1$ are solved completely.

Denote by $k$ the index with $\left|\beta^{(k)}\right|=\min \left|\beta^{(i)}\right|$ (the minimum is taken for $i=$ $1,2,3)$ and let $\{i, j\}=\{1,2,3\} \backslash\{k\}$. In the real case, we have to consider $k=1,2,3$ separately, but in the complex case, the only interesting case is when $\tau^{(k)}$ is the real conjugate of $\tau$ (cf. [21]). 
Applying Siegel's identity and some standard estimates, in the real case we obtain that, if $B=\max \left(\left|b_{1}\right|,\left|b_{2}\right|\right)$ is large enough,

$$
\left|b_{1} \log \right| \delta_{1}\left|+b_{2} \log \right| \delta_{2}|-\log | \delta_{3}||<\exp \left(c_{1}-c_{2} B\right) .
$$

In the complex case, if $\left|b_{1}\right|$ is not too small, then the corresponding inequality is

$$
\left|b_{1} \log \left(\delta_{1}\right)+b_{2} \log (-1)-\log \left(\delta_{2}\right)\right|<\exp \left(c_{1}-c_{2} B\right),
$$

where $\log$ denotes the principal value, $b_{2} \in Z$ with $\left|b_{2}\right| \leq 2\left|b_{1}\right|$ and $B=$ $\max \left(\left|b_{1}\right|,\left|b_{2}\right|\right)$. In both cases, $\delta_{1}, \delta_{2}, \delta_{3}$ are algebraic numbers in the Galois closure of $K$ depending on $\gamma$, the fundamental unit(s), $\tau$, and the indices $k, i, j$. Furthermore, $c_{1}, c_{2}, \ldots$ denote explicitly given positive constants.

Applying Baker's method and the better estimates of Waldschmidt [26] (for the sake of getting sharp constants), in both cases the respective linear forms can be estimated from below by a constant of the form

$$
\exp \left(-c_{3}\left(\log B+c_{4}\right)\right)
$$

Comparing the lower and upper estimates obtained for the linear forms (4), (5), we get an upper bound $B_{u}$ for $B$, which is about $10^{27}, 10^{28}$ in the cases we computed.

Dividing (4) and (5) by the coefficient of $b_{2}$ (in both cases), we obtain

$$
\left|b_{1} \theta+b_{2}-\xi\right|<c_{5} c_{6}^{-B}
$$

where $\theta, \xi$ are the quotients of two logarithms. This is the inequality to which the Baker-Davenport reduction method can be applied. For our case, a suitable version of the lemma is formulated in [11]. The essence of it is that, if we can find a good approximation of $\theta$, which does not approximate well $\xi$, then (6) has no solutions $b_{1}, b_{2}$ with

$$
\frac{c_{7}+\log B_{u}}{c_{8}}<B<B_{u},
$$

where $c_{7}, c_{8}$ have moderate values. Thus, we can reduce $B_{u}$ almost to its logarithm, and we get a much better upper bound for $B$. Repeating the reduction step three or four times (until the new bound is still less than the original one), we get quite a low bound for $B$, which, in our computation, was usually $\leq 12$. We remark that in the first reduction step it is necessary to use multiple precision arithmetic and to calculate the numerical values for $\theta$ and $\xi$ with an accuracy of about 100 digits.

Using the reduced bound for $B$, from (3) it is easy to derive a bound $y_{0}$ for $|y|$, and the procedure is completed by applying a fast algorithm of Pethö [21] which helps to find all solutions of (1) with $|y|<y_{0}$.

3. Computational Results. Using the above method, we solved index form equations (1) corresponding to cubic number fields with discriminants $-300 \leq D \leq$ 3137.

From a computational point of view, the totally real case is more interesting than the complex case. This is why we included more examples with positive discriminants. Further, let us remark that in the complex case, much more is known about the number of solutions of (1) than in the real case. To formulate the corresponding theorem, recall that two cubic forms $f_{1}, f_{2} \in Z[X, Y]$ are called equivalent if there exist integers $a_{1}, a_{2}, a_{3}, a_{4}$ with $a_{1} a_{4}-a_{2} a_{3}= \pm 1$ such that 
$f_{2}(X, Y)=f_{1}\left(a_{1} X+a_{2} Y, a_{3} X+a_{4} Y\right)$. A form $f(X, Y)=a X^{3}+b X^{2} Y+c X Y^{2}+$ $d Y^{3}$ is reversible if $a=d=1$ (cf. [6]).

Denote by $N$ the number of solutions of

$$
f(x, y)=1 \quad \text { in } x, y \in Z
$$

with the above $f$. Our equation (1) is precisely of the type (7), hence it is interesting to compare our results with those previously known about $N$. In the complex case, Delone [5] and Nagell [20] proved that $N \leq 5$, and that

$$
\begin{aligned}
& N=
\end{aligned}
$$

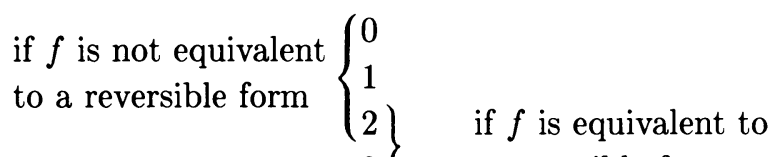

$$
\begin{aligned}
& 3 \text { a reversible form } \\
& 4 \text { if } D=-44 \text { and }-31 \\
& 5 \text { if } D=-23 \text {, }
\end{aligned}
$$

and there exist infinitely many inequivalent forms with $N=3$.

The corresponding assertion in the real case has not yet been verified, but our results make probable a conjecture of Pethö [22], namely that $N \leq 9$ and that

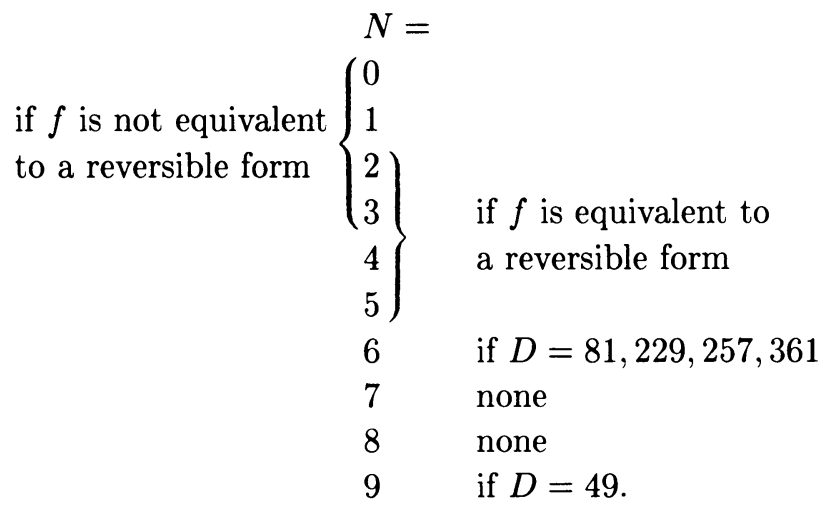

We remark that the numerical data of all power integral bases may have several applications, apart from using them in calculations in these fields. For example, Kovács [18] proved that the so-called canonical number systems of number fields (cf. [18]) are closely connected with power integral bases. Our data were used by Kovács and Pethö [19] to compute all canonical number systems in certain cubic number fields. Further, our table makes it possible to find several exceptional units in modules $\{1, \alpha\}\left(\alpha \in Z_{K}\right)$ of cubic number fields.

The numerical results are listed in the table below. For every example, the table contains the following data:

$$
D \quad\left(a_{2}, a_{1}, a_{0}\right) \quad\left(I_{3}, I_{2}, I_{1}, I_{0}\right) \quad\left(x_{1}, y_{1}\right),\left(x_{2}, y_{2}\right), \ldots,
$$

where $D$ is the discriminant of the field $K, f(x)=x^{3}+a_{2} x^{2}+a_{1} x+a_{0}$ is the defining polynomial of the generator element $\gamma$ of $K$ over $Q$. The index form is

$$
I(X, Y)=I_{3} X^{3}+I_{2} X^{2} Y+I_{1} X Y^{2}+I_{0} Y^{3},
$$

and the solutions of $(1)$ are $\left(x_{1}, y_{1}\right),\left(x_{2}, y_{2}\right), \ldots$ Obviously, if $(x, y)$ is a solution of $(1)$, then so is $(-x,-y)$, but we include only one of them in the table. If the 
given integral basis (corresponding to the index form) is different from $\left\{1, \gamma, \gamma^{2}\right\}$, and it has the form $\left\{1, w_{2}, w_{3}\right\}$ with

$$
w_{2}=\left(p_{0}+p_{1} \gamma+p_{2} \gamma^{2}\right) / p, \quad w_{3}=\left(q_{0}+q_{1} \gamma+q_{2} \gamma^{2}\right) / q
$$

then we also add the data

$$
w 2=\left(p_{0}, p_{1}, p_{2}\right) / p, \quad w 3=\left(q_{0}, q_{1}, q_{2}\right) / q .
$$

The input parameters (discriminant, defining polynomial of the generator element, integral basis, fundamental units) were taken in the real case from Ennola and Turunen [9], and in the complex case from Buchmann [3].

The computer program was developed in Fortran and was executed on the Siemens 7570P computer of the University of Düsseldorf. The execution time was about 60 seconds for every example.

TABLE

Real cubic fields

\begin{tabular}{rll}
$D$ & \multicolumn{1}{c}{$f(x)$} & \multicolumn{1}{c}{$I(X, Y)$} \\
49 & $(-1,-2,1)$ & $(1,2,-1,-1)$ \\
81 & $(0,-3,1)$ & $(1,0,-3,1)$ \\
148 & $(-1,-3,1)$ & $(1,2,-2,-2)$ \\
169 & $(-1,-4,-1)$ & $(1,2,-3,-5)$ \\
229 & $(0,-4,1)$ & $(1,0,-4,1)$ \\
& & \\
257 & $(-1,-4,3)$ & $(1,2,-3,-1)$ \\
316 & $(-1,-4,2)$ & $(1,2,-3,-2)$ \\
321 & $(-1,-4,1)$ & $(1,2,-3,-3)$ \\
361 & $(-1,-6,7)$ & $(1,2,-5,1)$ \\
404 & $(-1,-5,-1)$ & $(1,2,-4,-6)$ \\
469 & $(-1,-5,4)$ & $(1,2,-4,-1)$ \\
473 & $(0,-5,1)$ & $(1,0,-5,1)$ \\
564 & $(-1,-5,3)$ & $(1,2,-4,-2)$ \\
568 & $(-1,-6,-2)$ & $(1,2,-5,-8)$ \\
621 & $(0,-6,3)$ & $(1,0,-6,3)$ \\
697 & $(0,-7,5)$ & $(1,0,-7,5)$ \\
733 & $(-1,-7,8)$ & $(1,2,-6,1)$ \\
756 & $(0,-6,2)$ & $(1,0,-6,2)$ \\
761 & $(-1,-6,-1)$ & $(1,2,-5,-7)$ \\
785 & $(-1,-6,5)$ & $(1,2,-5,-1)$ \\
788 & $(-1,-7,-3)$ & $(1,2,-6,-10)$ \\
837 & $(0,-6,1)$ & $(1,0,-6,1)$ \\
892 & $(-1,-8,10)$ & $(1,2,-7,2)$ \\
940 & $(0,-7,4)$ & $(1,0,-7,4)$ \\
961 & $(-1,-10,8)$ & $(2,5,-1,-2)$ \\
& $w 2=(0,1,0) / 1$ & $w 3=(0,1,1) / 2$ \\
985 & $(-1,-6,1)$ & $(1,2,-5,-5)$ \\
993 & $(-1,-6,3)$ & $(1,2,-5,-3)$ \\
1016 & $(-1,-6,2)$ & $(1,2,-5,-4)$ \\
1076 & $(0,-8,6)$ & $(1,0,-8,6)$ \\
1101 & $(-1,-9,12)$ & $(1,2,-8,3)$ \\
1129 & $(0,-7,3)$ & $(1,0,-7,3)$ \\
1229 & $(-1,-7,6)$ & $(1,2,-6,-1)$ \\
1257 & $(-1,-8,9)$ & $(1,2,-7,1)$ \\
1300 & $(0,-10,10)$ & $(1,0,-10,10)$ \\
1345 & $(0,-7,1)$ & $(1,0,-7,1)$ \\
1369 & $(-1,-12,-11)$ & $(1,2,-11,-23)$ \\
& &
\end{tabular}

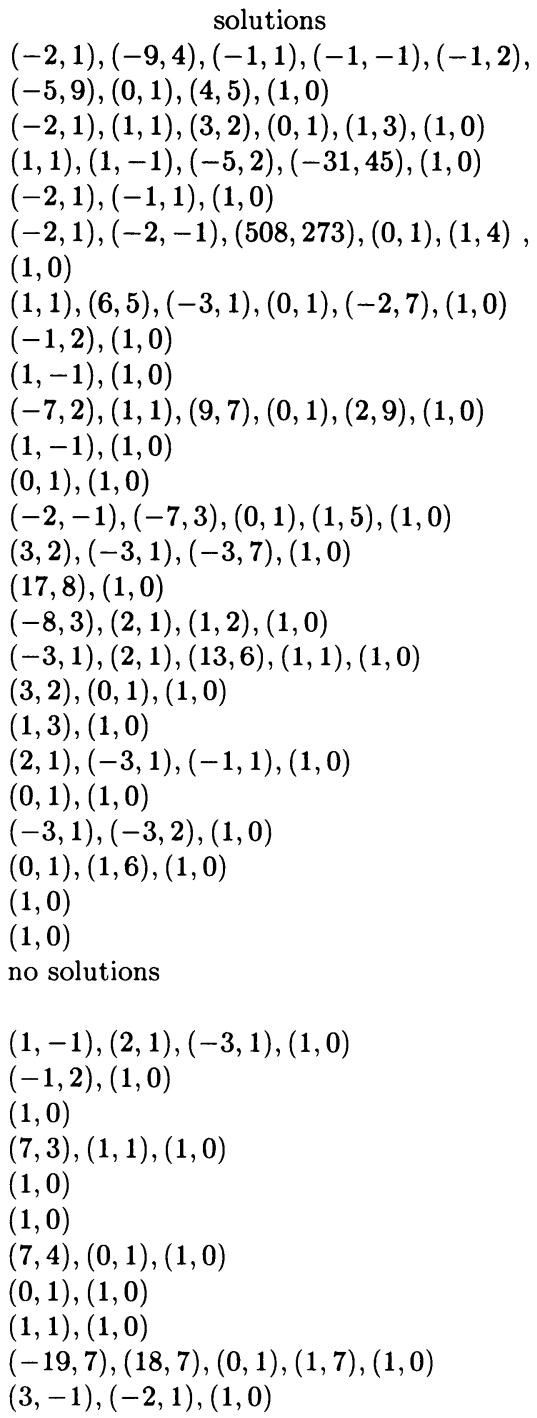




\section{TABLE (continued)}

\begin{tabular}{|c|c|c|c|}
\hline$D$ & $f(x)$ & $I(X, Y)$ & solutions \\
\hline 1373 & $(0,-8,5)$ & $(1,0,-8,5)$ & $(2,3),(1,0)$ \\
\hline 1384 & $(-1,-10,14)$ & $(1,2,-9,4)$ & $(25,14),(1,2),(1,0)$ \\
\hline 1396 & $(-1,-7,5)$ & $(1,2,-6,-2)$ & $(1,0)$ \\
\hline 1425 & $(-1,-8,-3)$ & $(1,2,-7,-11)$ & $(3,-1),(1,0)$ \\
\hline 1436 & $(0,-11,12)$ & $(1,0,-11,12)$ & $(5,2),(1,0)$ \\
\hline 1489 & $(-1,-10,-7)$ & $(1,2,-9,-17)$ & $(2,-1),(3,1),(3,-1),(-22,7),(1,0)$ \\
\hline 1492 & $(-1,-9,-5)$ & $(1,2,-8,-14)$ & $(3,-1),(1,0)$ \\
\hline 1509 & $(-1,-7,4)$ & $(1,2,-6,-3)$ & $(2,1),(1,0)$ \\
\hline 1524 & $(-1,-7,1)$ & $(1,2,-6,-6)$ & $(-1,1),(1,0)$ \\
\hline 1556 & $(-1,-9,11)$ & $(1,2,-8,2)$ & $(1,0)$ \\
\hline 1573 & $(-1,-7,2)$ & $(1,2,-6,-5)$ & $(2,1),(-13,18),(1,0)$ \\
\hline 1593 & $(0,-9,7)$ & $(1,0,-9,7)$ & $(-10,3),(5,2),(1,1),(1,0)$ \\
\hline 1620 & $(0,-12,14)$ & $(1,0,-12,14)$ & $(1,0)$ \\
\hline 1708 & $(-1,-8,-2)$ & $(1,2,-7,-10)$ & $(1,0)$ \\
\hline 1765 & $(-1,-11,16)$ & $(1,2,-10,5)$ & $(2,1),(1,0)$ \\
\hline 1772 & $\begin{array}{l}(-1,-12,8) \\
w 2=(0,1,0) / 1\end{array}$ & $\begin{array}{l}(2,5,-2,-3) \\
w 3=(0,1,1) / 2\end{array}$ & $(-8,3),(-2,3)$ \\
\hline 1825 & $(-1,-8,7)$ & $(1,2,-7,-1)$ & $(2,1),(0,1),(1,0)$ \\
\hline 1849 & $\begin{array}{l}(-1,-14,-8) \\
w 2=(0,1,0) / 1\end{array}$ & $\begin{array}{l}(2,5,-3,-8) \\
w 3=(0,1,1) / 2\end{array}$ & no solutions \\
\hline 1901 & $(-1,-9,-4)$ & $(1,2,-8,-13)$ & $(-42,13),(-3,2),(1,0)$ \\
\hline 1929 & $(-1,-10,13)$ & $(1,2,-9,3)$ & $(2,1),(1,0)$ \\
\hline 1937 & $(-1,-8,-1)$ & $(1,2,-7,-9)$ & $(-1,1),(1,0)$ \\
\hline 1940 & $(0,-8,2)$ & $(1,0,-8,2)$ & $(-3,1),(1,4),(1,0)$ \\
\hline 1944 & $(0,-9,6)$ & $(1,0,-9,6)$ & $(1,0)$ \\
\hline 1957 & $(-1,-9,10)$ & $(1,2,-8,1)$ & $(-4,1),(2,1),(0,1),(1,0)$ \\
\hline 2021 & $(0,-8,1)$ & $(1,0,-8,1)$ & $(-26,9),(0,1),(1,8),(1,0)$ \\
\hline 2024 & $(-1,-10,-6)$ & $(1,2,-9,-16)$ & $(1,0)$ \\
\hline 2057 & $(0,-11,11)$ & $(1,0,-11,11)$ & $(1,1),(1,0)$ \\
\hline 2089 & $\begin{array}{l}(0,-13,4) \\
w 2=(0,1,0) / 1\end{array}$ & $\begin{array}{l}(2,3,-5,-2) \\
w 3=(0,1,1) / 2\end{array}$ & no solutions \\
\hline 2101 & $(-1,-11,-8)$ & $(1,2,-10,-19)$ & $(-2,1),(1,0)$ \\
\hline 2177 & $(-1,-8,5)$ & $(1,2,-7,-3)$ & $(2,1),(1,0)$ \\
\hline 2213 & $(-1,-13,-12)$ & $(1,2,-12,-25)$ & $(-2,1),(1,0)$ \\
\hline 2233 & $(-1,-8,1)$ & $(1,2,-7,-7)$ & $(-1,1),(1,0)$ \\
\hline 2241 & $(0,-9,5)$ & $(1,0,-9,5)$ & $(8,3),(1,0)$ \\
\hline 2296 & $(-1,-14,-14)$ & $(1,2,-13,-28)$ & $(-9,4),(1,0)$ \\
\hline 2300 & $(-1,-8,2)$ & $(1,2,-7,-6)$ & $(-7,2),(1,0)$ \\
\hline 2349 & $(0,-12,13)$ & $(1,0,-12,13)$ & $(8,3),(1,0)$ \\
\hline 2505 & $(-1,-10,-5)$ & $(1,2,-9,-15)$ & $(1,0)$ \\
\hline 2557 & $(-1,-9,-2)$ & $(1,2,-8,-11)$ & $(1,0)$ \\
\hline 2589 & $\begin{array}{l}(-1,-14,12) \\
w 2=(0,1,0) / 1\end{array}$ & $\begin{array}{l}(2,5,-3,-3) \\
w 3=(0,1,1) / 2\end{array}$ & $(1,1)$ \\
\hline 2597 & $(-1,-9,8)$ & $(1,2,-8,-1)$ & $(2,1),(-4,1),(0,1),(1,0)$ \\
\hline 2636 & $\begin{array}{l}(0,-14,4) \\
w 2=(0,1,0) / 1\end{array}$ & $\begin{array}{l}(2,0,-7,1) \\
w 3=(0,0,1) / 2\end{array}$ & $(-2,1),(0,1)$ \\
\hline 2673 & $(0,-9,3)$ & $(1,0,-9,3)$ & $(1,3),(1,0)$ \\
\hline 2677 & $(0,-10,7)$ & $(1,0,-10,7)$ & $(1,0)$ \\
\hline 2700 & $(0,-15,20)$ & $(1,0,-15,20)$ & $(1,0)$ \\
\hline 2708 & $(-1,-11,-7)$ & $(1,2,-10,-18)$ & $(1,0)$ \\
\hline 2713 & $(0,-13,15)$ & $(1,0,-13,15)$ & $(-49,12),(4,3),(1,0)$ \\
\hline 2777 & $(-1,-14,23)$ & $(1,2,-13,9)$ & $(-5,1),(2,1),(17,8),(1,1),(1,0)$ \\
\hline 2804 & $(-1,-9,-1)$ & $(1,2,-8,-10)$ & $(-1,1),(1,0)$ \\
\hline 2808 & $(0,-9,2)$ & $(1,0,-9,2)$ & $(1,0)$ \\
\hline 2836 & $(-1,-9,7)$ & $(1,2,-8,-2)$ & $(1,0)$ \\
\hline 2857 & $(-1,-10,11)$ & $(1,2,-9,1)$ & $(-21,5),(2,1),(1,0),(0,1)$ \\
\hline 2917 & $(-1,-13,20)$ & $(1,2,-12,7)$ & $(2,1),(1,0)$ \\
\hline
\end{tabular}




\section{TABLE (continued)}

\begin{tabular}{cl}
$D$ & \multicolumn{1}{c}{$f(x)$} \\
2981 & $(-1,-11,14)$ \\
2993 & $(-1,-12,17)$ \\
3021 & $(-1,-9,6)$ \\
3028 & $(0,-10,6)$ \\
3137 & $(0,-11,9)$
\end{tabular}

D

$-23 \quad(0,-1,1)$

$-31 \quad(0,1,1)$

$-44 \quad(1,-1,1)$

$-59 \quad(0,2,1)$

$-76 \quad(0,-2,2)$

$-83 \quad(1,1,2)$

$-87 \quad(1,-2,-3)$

$-104(0,-1,2)$

$-107 \quad(1,3,2)$

$-108 \quad(0,0,2)$

$-116 \quad(1,0,2)$

$-135 \quad(0,-3,3)$

$-139 \quad(1,1,-2)$

$-140 \quad(0,2,2)$

$-152(1,-2,2)$

$-172 \quad(1,-1,-3)$

$-175 \quad(1,2,3)$

$-199 \quad(1,-4,3)$

$-200 \quad(1,2,-2)$

$-204 \quad(1,1,3)$

$-211 \quad(0,-2,3)$

$-212 \quad(1,4,2)$

$-216 \quad(0,3,2)$

$-231 \quad(1,0,-3)$

$-239 \quad(0,-1,3)$

$-243(0,0,3)$

$-244 \quad(1,-4,-6)$

$-247 \quad(0,1,3)$

$-255 \quad(1,0,3)$

$-268 \quad(1,-3,-5)$

$-283 \quad(0,4,1)$

$-300 \quad(1,-3,3)$
$I(X, Y)$

$(1,2,-10,3)$

$(1,2,-11,5)$

$(1,2,-8,-3)$

$(1,0,-10,6)$

$(1,0,-11,9)$

solutions

\section{Complex cubic fields}

\begin{tabular}{ll}
\multicolumn{1}{c}{$I(X, Y)$} & \multicolumn{1}{c}{ solutions } \\
$(1,0,-1,1)$ & $(-1,1),(0,1),(1,0),(1,1),(4,-3)$ \\
$(1,0,1,1)$ & $(-2,3),(0,1),(1,-1),(1,0)$ \\
$(1,-2,0,2)$ & $(-47,56),(1,-1),(1,0),(1,1)$ \\
$(1,0,2,1)$ & $(0,1),(1,-2),(1,0)$ \\
$(1,0,-2,2)$ & $(-23,13),(1,0),(1,1)$ \\
$(1,-2,2,1)$ & $(0,1),(1,0)$ \\
$(1,-2,-1,-1)$ & $(0,-1),(1,0)$ \\
$(1,0,-1,2)$ & $(-3,2),(1,0)$ \\
$(1,-2,4,-1)$ & $(0,-1),(1,0),(2,7)$ \\
$(1,0,0,2)$ & $(-1,1),(1,0)$ \\
$(1,-2,1,2)$ & $(1,0)$ \\
$(1,0,-3,3)$ & $(-2,1),(1,0),(1,1)$ \\
$(1,-2,2,-3)$ & $(1,0),(2,1)$ \\
$(1,0,2,2)$ & $(1,-1),(1,0)$ \\
$(1,-2,-1,4)$ & $(1,0)$ \\
$(1,-2,0,-2)$ & $(1,0)$ \\
$(1,-2,3,1)$ & $(0,1),(1,0)$ \\
$(1,-2,-3,7)$ & $(1,0),(2,1)$ \\
$(1,-2,3,-4)$ & $(1,0)$ \\
$(1,-2,2,2)$ & $(1,0)$ \\
$(1,0,-2,3)$ & $(1,0),(2,-1)$ \\
$(1,-2,5,-2)$ & $(1,0),(1,2)$ \\
$(1,0,3,2)$ & $(1,0)$ \\
$(1,-2,1,-3)$ & $(-2,-1),(1,0)$ \\
$(1,0,-1,3)$ & $(-5,3),(1,0)$ \\
$(1,0,0,3)$ & $(1,0)$ \\
$(1,-2,-3,-2)$ & $(1,0)$ \\
$(1,0,1,3)$ & $(-1,1),(1,0)$ \\
$(1,-2,1,3)$ & $(1,-1),(1,0)$ \\
$(1,-2,-2,-2)$ & $(1,0),(3,1)$ \\
$(1,0,4,1)$ & $(0,1),(1,-4),(1,0)$ \\
$(1,-2,-2,6)$ & $(1,0)$ \\
$(0,1)$
\end{tabular}

After the complete list of cubic fields with discriminants $-300 \leq D \leq 3137$ we give some more examples which may be of interest.

\begin{tabular}{rlll}
$D$ & \multicolumn{1}{c}{$f(x)$} & \multicolumn{1}{c}{$I(X, Y)$} & \multicolumn{1}{c}{ solutions } \\
22356 & $(0,-36,60)$ & $(2,0,-18,15)$ & $(1,1)$ \\
& $w 2=(0,1,0) / 1$ & $w 3=(0,0,1) / 2$ & \\
22356 & $(0,-18,6)$ & $(1,0,-18,6)$ & $(1,3),(1,0)$ \\
22356 & $(0,-36,78)$ & $(1,0,-36,78)$ & $(485,117),(1,0)$ \\
677329 & $(1,-274,61)$ & $(11,-14,-19,11)$ & no solutions \\
& $w 2=(0,1,0) / 1$ & $w 3=(-1,-4,1) / 11$ &
\end{tabular}


Mathematisches Institut

Universität Düsseldorf

Universitätsstrasse 1

4000 Düsseldorf 1

Federal Republic of Germany

1. G. ARchinard, "Extensions cubiques cycliques de $Q$ dont l'anneau des entiers est monogène," Enseign. Math., v. 20, 1974, pp. 179-203.

2. A. BAKER \& H. DAVEnPORT, "The equations $3 x^{2}-2=y^{2}$ and $8 x^{2}-7=z^{2}$," Quart. J. Math. Oxford Ser. (2), v. 20, 1969, pp. 129-137.

3. J. BUCHMANN, "A generalization of Voronoi's unit algorithm. I," J. Number Theory, v. 20, 1985, pp. 177-191.

4. D. S. DUMmit \& H. Kisilevsky, "Indices in cyclic cubic fields," in Number Theory and Algebra, Academic Press, New York, 1977, pp. 29-42.

5. B. N. DELONE, "Über die Darstellung der Zahlen durch die binären kubischen Formen von negativer Diskriminante," Math. Z., v. 31, 1930, pp. 1-26.

6. B. N. DELONE \& D. K. FADDEEV, The Theory of Irrationalities of the Third Degree, Transl. Math. Monographs, no. 10, Amer. Math. Soc., Providence, R.I., 1964.

7. W. J. Ellison, Recipes for Solving Diophantine Problems by Baker's Method, Sém. Theorie Nombres, Année 1970-1971, exp. no. 11.

8. W. J. Ellison, F. Ellison, J. Pesek, C. E. Stahl \& D. S. Stall, "The Diophantine equation $y^{2}+k=x^{3}, " J$. Number Theory, v. 4, 1972, pp. 107-117.

9. V. ENNola \& R. TURUnen, "On totally real cubic fields," Math. Comp., v. 44, 1985, pp. 495-518.

10. U. Fincke \& M. Pohst, A Procedure for Determining Algebraic Integers of Given Norm, EUROCAL 83, Lecture Notes in Computer Science, vol. 162, Springer-Verlag, Berlin and New York, 1983, pp. 194-202.

11. I. GAÁL, "On the resolution of inhomogeneous norm form equations in two dominating variables," Math. Comp., v. 51, 1988, pp. 359-373.

12. M. N. Gras, Sur les Corps Cubiques Cycliques dont l'Anneau des Entiers est Monogène, Publ. Math. Fac. Sci. Besançon, 1973.

13. M. N. GRAS, Lien Entre le Groupe des Unités et la Monogèneité des Corps Cubiques Cycliques, Sém. Théorie Nombr. Besançon, Année 1975-76.

14. K. GYÖRY, "Sur les polynomes à coefficients entiers et de discriminant donné. III," Publ. Math. (Debrecen), v. 23, 1976, pp. 141-165.

15. K. GYÖRY, Résultats Effectives sur la Représentation des Entiers par des Formes Décomposables, Queen's Papers in Pure and Applied Math., No. 56, Kingston, Canada, 1980.

16. K. GYÖRY, "Bounds for the solutions of norm form, discriminant form and index form equations in finitely generated integral domains," Acta Math. Hungar., v. 42, 1983, pp. 45-80.

17. K. GYÖRY \& Z. Z. PAPP, "On discriminant form and index form equations," Studia Sci. Math. Hungar., v. 12, 1977, pp. 47-60.

18. B. KovÁCs, "Canonical number systems in algebraic number fields," Acta Math. Acad. Sci. Hungar., v. 37, 1981, pp. 405-407.

19. В. KOVÁcS \& A. РетнÖ, "Number systems in integral domains, especially in orders of algebraic number fields," in preparation.

20. T. NAGELL, "Darstellung ganzer Zahlen durch binäre kubische Formen mit negativer Diskriminante," Math. Z., v. 28, 1928, pp. 10-29.

21. A. РЕтнÖ, "On the resolution of Thue inequalities," J. Symbolic Comp., v. 4, 1987, pp. 103-109.

22. A. Ретнӧ, On the Representation of 1 by Binary Cubic Forms with Positive Discriminant, in Proceedings Journées Arithmétique, Ulm. (To appear.)

23. А. Ретнӧ \& R. Schulenberg, "Effektives Lösen von Thue Gleichungen," Publ. Math. (Debrecen), v. 34, 1987, pp. 189-196.

24. R. P. Steiner, "On Mordell's equation $y^{2}-k=x^{3}$ : A problem of Stolarsky," Math. Comp., v. 46, 1986, pp. 703-714.

25. L. A. Trelina, "On the greatest prime factor of an index form," Dokl. Akad. Nauk BSSR, v. 21, 1977, pp. 975-976. (Russian)

26. M. WA LDSChmidt, "A lower bound for linear forms in logarithms," Acta Arith., v. 37, 1980, pp. 257-283. 